\title{
Thrombolytic Therapy and Aspiration of Clots were Effective in the Removal of a Transient Inferior Vena Cava (IVC) Filter that Captured a Large Thrombus
}

\author{
Norihito Takahashi, Kikuo Isoda, Masaru Hiki, Shinichiro Fujimoto and Hiroyuki Daida
}

\begin{abstract}
A 37-year-old woman presented at 37 weeks of gestation with a history of heaviness in her left leg. Enhanced computed tomography revealed a left extensive ileofemoral thrombus, and we employed a transient inferior vena cava (IVC) filter (t-IVCF) before delivery. Although we attempted to remove the t-IVCF on the day after delivery, a large thrombus was captured in the filter. We therefore performed thrombolytic therapy for one week, but a few small clots remained within the t-IVCF. We were ultimately able to remove the tIVCF under constant negative pressure by aspiration from a side-hole of the sheath. We herein describe the effectiveness of this aspiration technique in our case.
\end{abstract}

Key words: deep vein thrombosis, pregnancy, IVC filter, thrombolytic therapy, aspiration

(Intern Med 56: 1369-1373, 2017)

(DOI: 10.2169/internalmedicine.56.8175)

\begin{abstract}
Introduction
Pregnancy and postpartum are periods of increased risk of deep vein thrombosis (DVT) and subsequent pulmonary embolism (PE) due to hemodynamic changes and changes in the coagulation and fibrinolytic systems (1). In the management of thromboembolic events in pregnancy, anticoagulation treatment with heparin needs to be performed immediately once DVT is diagnosed. However, anticoagulation therapy increases the risk of peripartum hemorrhaging during delivery and is therefore ceased 12 hours before labor (2). Because decreasing the dose of heparin increases the risk of $\mathrm{PE}$, transient inferior vena cava filter (t-IVCF) placement is a suitable treatment for pregnant women with a high risk of PE. In our case, a t-IVCF worked well for the prophylaxis of PE, but several clots did not dissolve and were captured within the filter after thrombolytic therapy. We were ultimately able to remove the t-IVCF under constant negative pressure by aspiration from a side-hole of the sheath, and no $\mathrm{PE}$ or other filter-related complications were observed.

We herein describe the effectiveness of the aspiration of clots to ameliorate several clots captured in a t-IVCF after
\end{abstract}

thrombolytic therapy.

\section{Case Report}

A 37-year-old woman presented at 34 weeks of gestation with a history of pain and heaviness in her left leg. A clinical examination showed a swollen left leg. Doppler venogram revealed left ileofemoral thrombus, and the patient was started on anticoagulant therapy for DVT at a clinic. Except for pregnancy, she had no risk factors for DVT, including no abnormalities in the protein C/protein S anticoagulant system, anti-cardiolipin antibody, anti-nuclear antibody or lack of antithrombin. The patient wanted to deliver her baby at our hospital, so she was admitted to our institute at 37 weeks of gestation.

Although her activated partial thromboplastin time had been maintained to within 45-70 seconds (1.5-2.3 times control) using unfractionated heparin for 25 days, an enhanced computed tomography (CT) showed a left extensive ileofemoral thrombus (Fig. 1), and we employed a t-IVCF (Toray Medical, Tokyo, Japan) at the suprarenal position shortly before delivery. The filter was inserted percutaneously through a sheath via the right internal jugular vein

Department of Cardiovascular Medicine, Juntendo University Graduate School of Medicine, Japan

Received for publication August 24, 2016; Accepted for publication December 9, 2016

Correspondence to Dr. Kikuo Isoda, kisoda@juntendo.ac.jp 


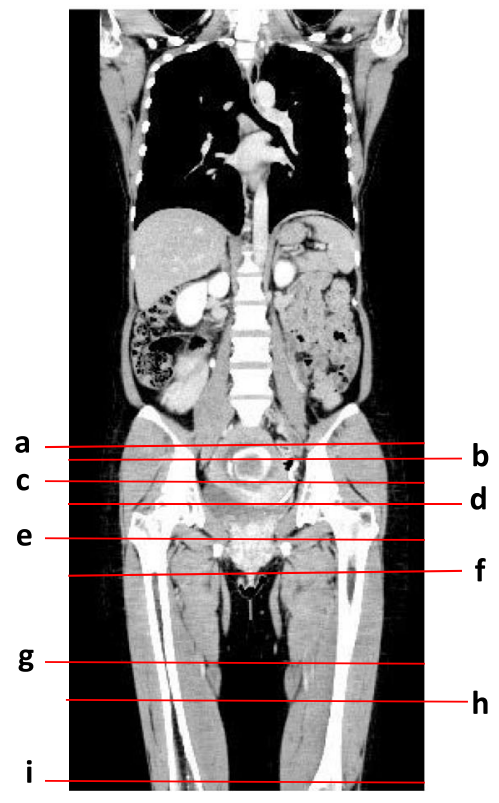

A
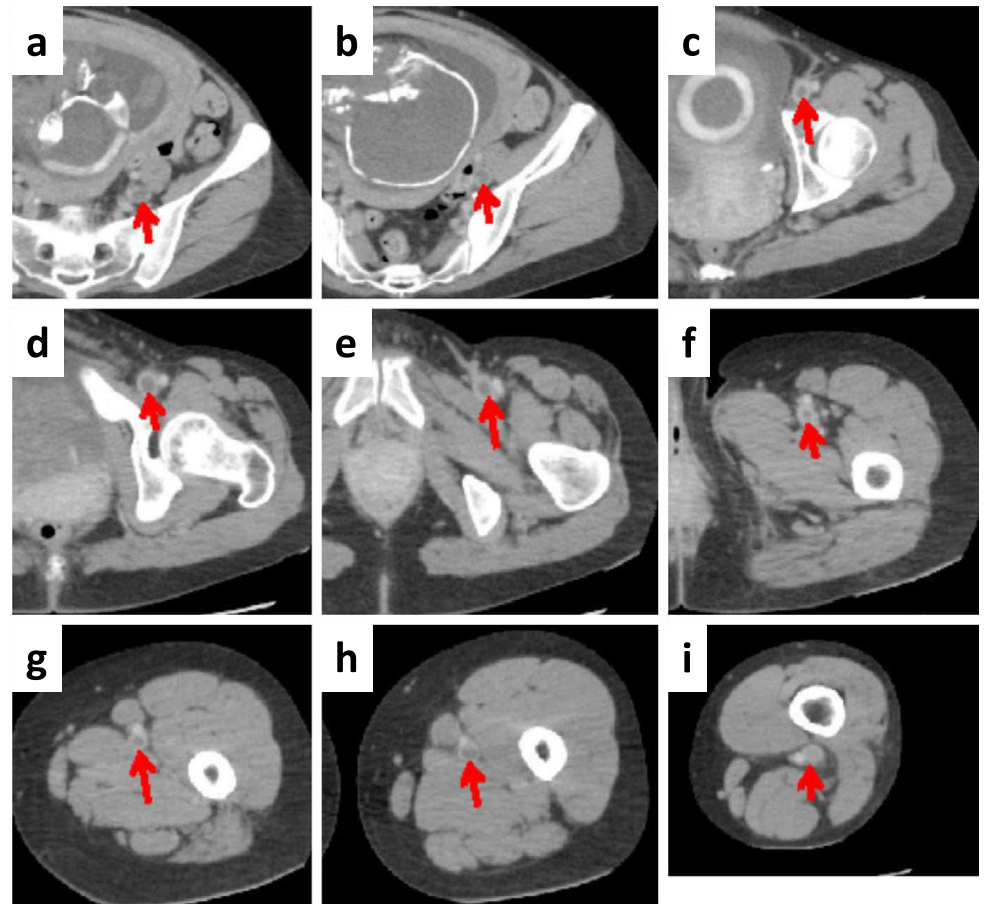

B

Figure 1. An enhanced CT showed a left extensive ileofemoral thrombus at 37 weeks of gestation. The lines (a-i) in the coronal section (A) show the levels of transverse sections (a-i in panels B). The arrows indicate a large thrombus in the vein.

A
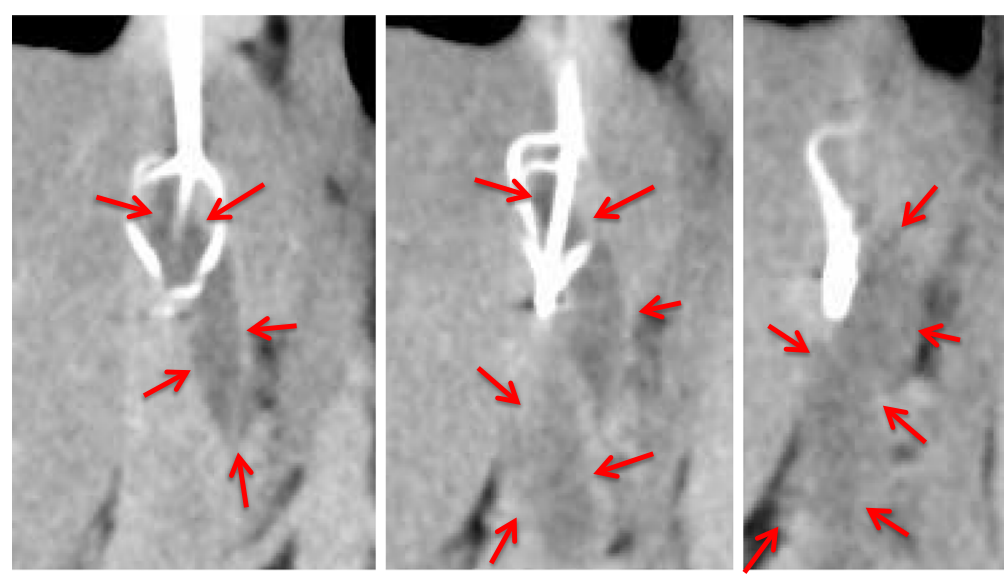

B

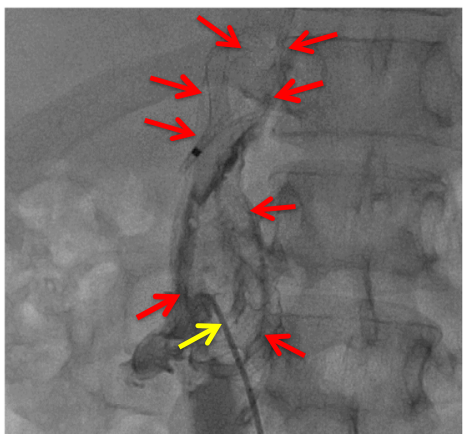

Figure 2. A: Continuous images of an enhanced CT after delivery showed that a large thrombus had been captured in the transient inferior vena cava filter (t-IVCF) (red arrows). B: Although we performed mechanical thrombus fragmentation using a pigtail catheter (yellow arrow), the size of thrombus did not change (red arrows).

without complications. The next morning, a healthy baby was delivered, and we attempted to remove the t-IVCF the day after delivery. However, an enhanced CT showed that a large thrombus had been captured in the filter (Fig. 2A red 

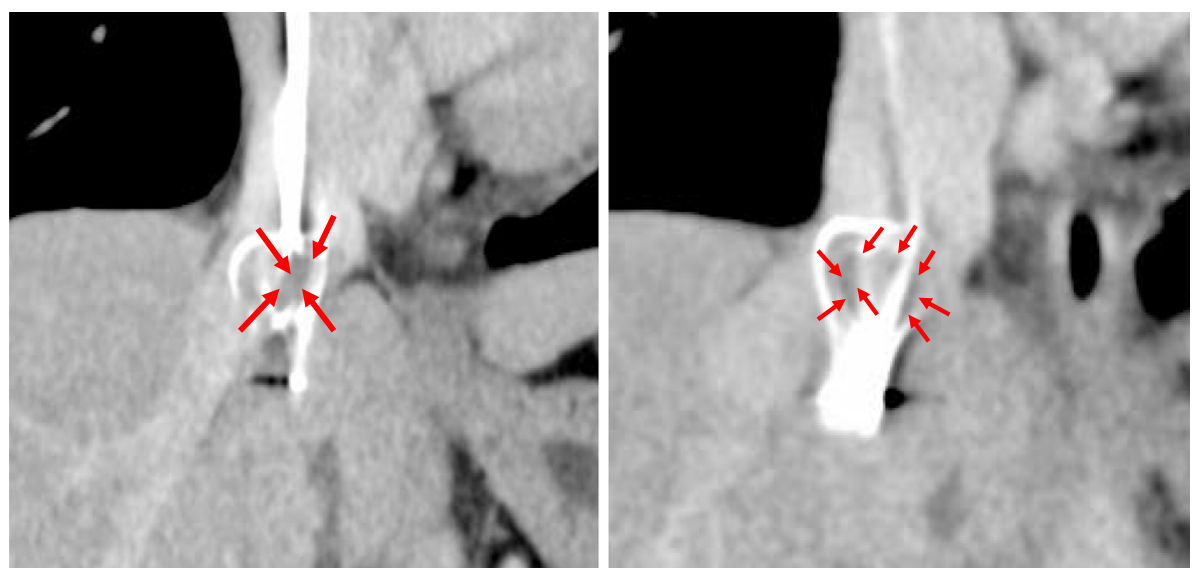

Figure 3. Continuous images of an enhanced CT one week after thrombolytic therapy revealed that the thrombus had shrunk significantly, but a few clots remained in the filter (red arrows).

A

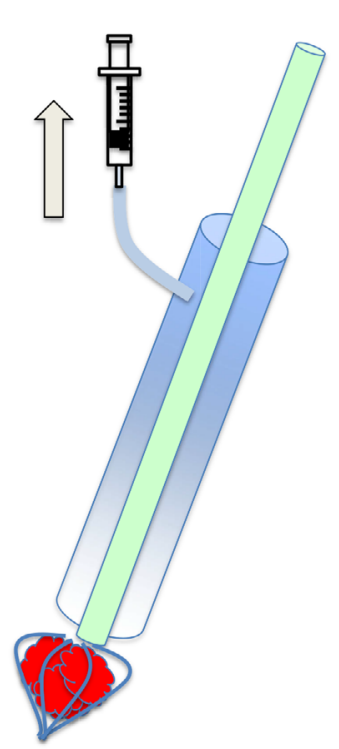

B

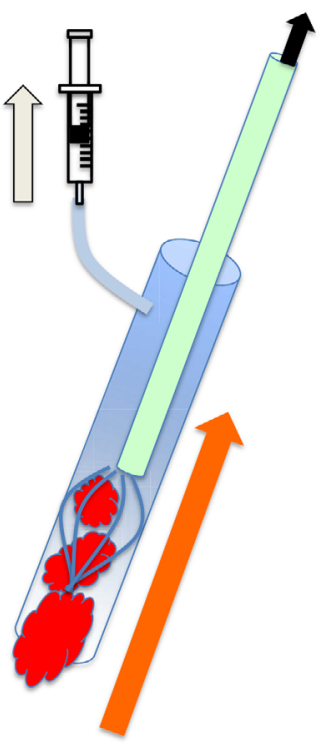

C

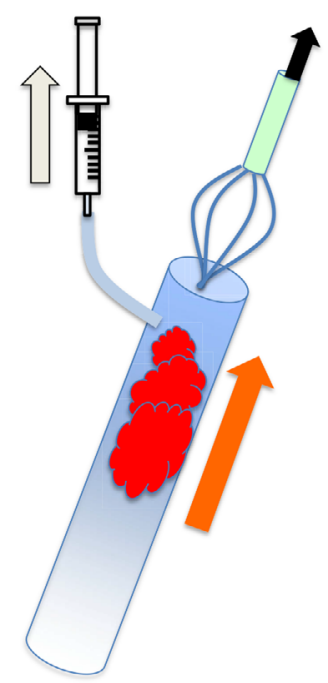

Figure 4. A schematic illustration of the aspiration procedure. After starting aspiration from a side-hole of the sheath, the t-IVCF containing clots was pulled back into the sheath (A). The clots within the t-IVCF were then squeezed out $(B)$ and aspirated into the sheath $(C)$.

arrows), believed to be due to DVT in a lower extremity.

Although we performed catheter thrombus fragmentation using a pigtail catheter (Fig. 2B yellow arrow), the size of the thrombus did not change (Fig. 2B red arrows). We therefore started thrombolytic therapy by the systemic administration of urokinase (480,000 units/day) for 1 week, and an enhanced CT revealed that the thrombus had shrunk significantly, although a few small clots remained within the tIVCF (Fig. 3 red arrows). We wanted to collect as many clots from the t-IVCF as possible to avoid the occurrence of $\mathrm{PE}$, as her clots were too hard to dissolve using thrombolysis or catheter thrombus fragmentation. We then removed the t-IVCF under constant negative pressure by aspiration from a side-hole of the sheath (Fig. 4). The aspirated clots con- sisted of a combination of fresh (Fig. 5A blue arrows and $\mathrm{B}$ ) and old organized thrombi (Fig. 5A yellow arrows and $\mathrm{C}$ ). No $\mathrm{PE}$ or other filter-related complications were observed.

\section{Discussion}

Pregnancy and postpartum are periods of increased risk of DVT and subsequent PE due to hemodynamic changes and changes in the coagulation and fibrinolytic systems (1). In the management of thromboembolic events in pregnancy, anticoagulation treatment with heparin needs to be performed immediately once DVT is diagnosed. However, anticoagulation therapy increases the risk of peripartum hemorrhaging 


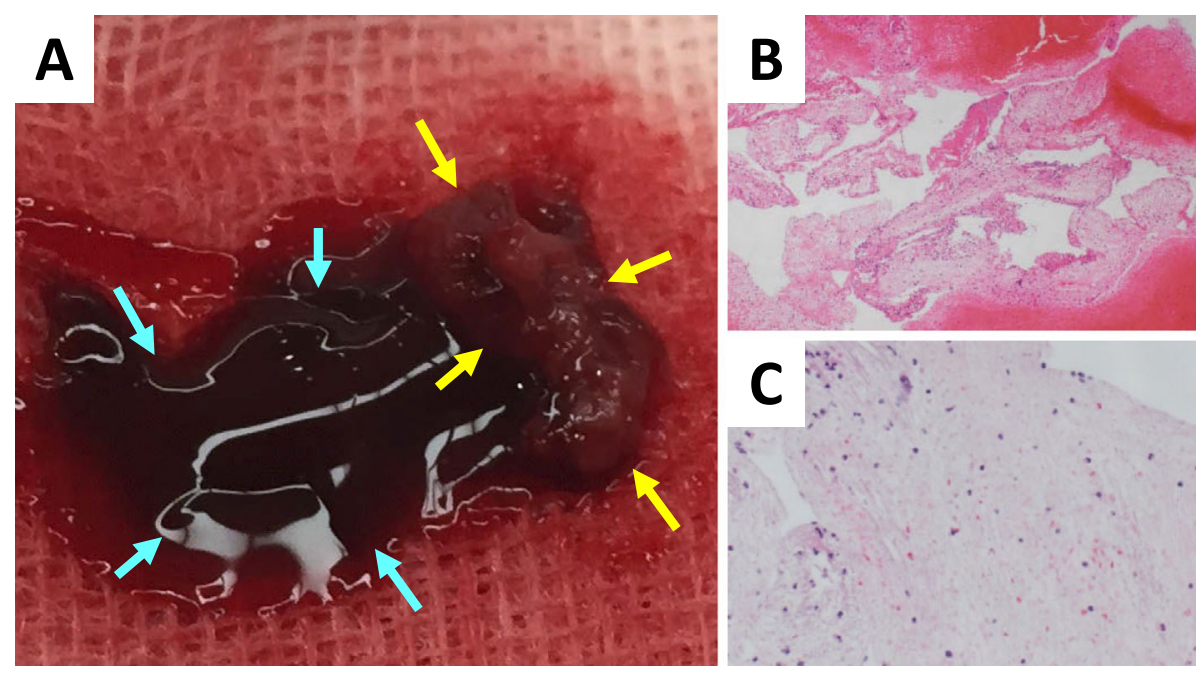

Figure 5. The aspirated clots consisted of a combination of fresh (A blue arrows and B) and old organized thrombi (A yellow arrows and C).

during delivery and is therefore ceased 12 hours before labor (2). Because decreasing the dose of heparin increases the risk of PE, t-IVCF placement is a suitable treatment for pregnant women with a high risk of PE. We therefore employed a t-IVCF at the suprarenal position shortly before delivery in our patient. We selected a t-IVCF because pregnancy involves only a temporary risk of DVT, and the patient professed a desire to have the filter removed after delivery. Suprarenal placement of a t-IVCF is suitable for pregnant women to avoid contact between the gravid uterus and the filter and to protect against thrombi development in ovarian veins (3). Furthermore, no severe complications (including renal dysfunction) have been reported with this placement site.

A previous report using the same kind of t-IVCF in 33 patients found that large thromobi were captured in the $\mathrm{t}$ IVCF in 4 patients $(12.1 \%)$ who then received catheterdirected thrombolytic therapy (4). In that report, a thrombus in 1 patient $(3.0 \%)$ did not dissolve, and it was difficult to remove the t-IVCF with it (4). Another recent case report using the same t-IVCF (Toray Medical, Tokyo, Japan) caught a wide thrombus after Caesarean section, and the authors performed catheter thrombus fragmentation with fibrinolysis just before the removal of the t-IVCF (5). Given these present and previous findings, we must be aware of the probable presence of a large thrombus beneath the tIVCF before attempting its removal.

In our patient, a large thrombus was captured in the tIVCF, considered to be due to DVT in a lower extremity. These findings suggested that the t-IVCF worked well for the prophylaxis of $\mathrm{PE}$, and checking for thrombus in a $\mathrm{t}$ IVCF should be performed before its removal. Although the catheter-directed thrombus fragmentation technique did not work well in the present patient, we were still able to make a few channels in the original thrombus, thereby facilitating thrombolysis with a fibrinolytic agent. An enhanced CT one week after thrombolytic therapy revealed that the thrombus had shrunk significantly.

One of the novel findings in our case is that aspiration from a side-hole of the sheath was very effective for the collection of the small clots still present within the t-IVCF. Although the incidence of hemodynamically unstable PE might be low without aspiration, we believe several benefits of this technique should be considered for the removal of t-IVCF containing clots. First, venotomy in her jugular vein was avoided by using this technique. Indeed, a previous report showed that a thrombus had not dissolved and was captured within the t-IVCF after catheter-directed thrombolytic therapy, and the thrombus inside the filter was removed along with the catheter through a venotomy in the jugular vein in the insertion site without protection (4). Second, we were able to collect almost all of the clots from the t-IVCF, and no PE or other filter-related complications were observed. If the clots in our case had escaped and induced a small PE, the PE might have been resistant to therapy because her organized clots were too hard to dissolve using thrombolysis or catheter thrombus fragmentation. Third, this technique allowed us to obtain histological samples of the clots. Other investigators detected small thrombi in the t-IVCFs after removal, and almost all were freshly formed, as seen in their pathological findings, suggesting that they had formed because of the filters themselves (3). However, Tardy et al. (6) suggested that early IVCF thrombosis was likely to be secondary to DVT in the lower extremities. They also suggested that it might be difficult to differentiate a native thrombus formed within an IVCF from a thrombus captured in the filter from a distal source using image findings alone (6). We believe that a histopathological analysis is needed to identify such differences. In our case, the histological findings demonstrated that the clots were relatively old, organized thrombi, indicating that they had been present before the t-IVCF insertion. We therefore concluded that the organized thrombi were too hard to break up using catheter thrombus fragmentation. 
We demonstrate the effectiveness of both thrombolytic therapy and the aspiration technique for the treatment of a large thrombus captured in a t-IVCF. To our knowledge, this aspiration technique has not been used for the removal of a t-IVCF that captured a few clots.

The authors state that they have no Conflict of Interest (COI).

\section{References}

1. Kocher M, Krcova V, Cerna M, Prochazka M. Retrievale Günther Tulip Vena Cava Filter in the prevention of pulmonary embolism in patients with acute deep venous thrombosis in perinatal period. Eur J Radiol 70: 165-169, 2009.

2. Bates SM. Treatment and prophylaxis of venous thromboembolism during pregnancy. Thromb Res 108: 97-106, 2002.

3. Liu Y, Sun Y, Zhang S, Jin X. Placement of a retrivale inferior vena cava filter for deep venous thrombosis in term pregnancy. $\mathbf{J}$ Vasc Surg 55: 1042-1047, 2012.

4. Miyahara T, Miyata T, Shigematsu K, et al. Clinical outcome and complications of temporary inferior vena cava filter placement. J Vasc Surg 44: 620-624, 2006.

5. Horie K, Suzuki H, Ohkuchi A, Matsubara S, Ikemoto T, Suzuki M. Thrombus just beneath a retriaval inferior vena cava filter in a pregnant woman with deep vein thrombosis: Its removal requiring catheter thrombus fragmentation with fibrinolysis. J Obstet Gynaecol Res 40: 590-594, 2014.

6. Tardy B, Mismetti P, Page Y, et al. Symptomatic inferior vena cava filter thrombosis: clinical study of 30 consecutive cases. Eur Respir J 9: 2012-2016, 1996.

The Internal Medicine is an Open Access article distributed under the Creative Commons Attribution-NonCommercial-NoDerivatives 4.0 International License. To view the details of this license, please visit (https://creativecommons.org/licenses/ by-nc-nd/4.0/).

(C) 2017 The Japanese Society of Internal Medicine http://www.naika.or.jp/imonline/index.html 